\title{
PROCESSING AND DISPLAY OF SPECTRAL LINE ABSORPTION MAPS
}

Melvyn Wright

Radio Astronomy Laboratory

U.C. Berkeley, California, U.S.A.

\section{ABSTRACT}

We describe the processing and display of aperture synthesis maps of the H I absorption against the radio source Cas $A$. Then data were obtained with the NRAO interferometer and constitute 384 narrow band maps of Cas A combined to produce a picture of the optical depth and mean velocity of the $\mathrm{HI}$ absorption due to the Perseus arm.

Both digital and analogue data techniques have been used to Fourier transform, combine and display the data and the merits and usefulness of each are described. 